\section{Case Reports in Gastroenterology}

Case Rep Gastroenterol 2016;10:108-114

This article is licensed under the Creative Commons Attribution-NonCommercial 4.0 International License (CC BY-NC) (http://www.karger.com/Services/OpenAccessLicense). Usage and distribution for commercial purposes requires written permission.

\title{
Orbital Pseudotumor as a Rare Extrahepatic Manifestation of Hepatitis C Infection
}

\author{
Benjamin Misselwitz ${ }^{a}$ Jana Epprecht ${ }^{a} \quad$ Joachim Mertens $^{a}$ \\ Luc Biedermann $^{a} \quad$ Michael Scharl $^{a} \quad$ Eugenia Haralambieva ${ }^{b}$ \\ Andreas Lutterotti ${ }^{c} \quad$ Konrad P. Weber ${ }^{c, d} \quad$ Beat Müllhaupt ${ }^{a}$ \\ Karla Chaloupka ${ }^{d}$ \\ ${ }^{a}$ Division of Gastroenterology and Hepatology, University Hospital Zurich, University of \\ Zurich, Zurich, Switzerland; ${ }^{b}$ Division of Pathology, University Hospital Zurich, University \\ of Zurich, Zurich, Switzerland; ' Division of Neurology, University Hospital Zurich, \\ University of Zurich, Zurich, Switzerland; ${ }^{d}$ Division of Ophthalmology, University Hospital \\ Zurich, University of Zurich, Zurich, Switzerland
}

\section{Keywords}

Hepatitis C - Extrahepatic manifestation - Lymphocytic dacryoadenitis · Orbital pseudotumor . Idiopathic orbital inflammation

\begin{abstract}
Hepatitis $C$ is frequently accompanied by immune-related extrahepatic manifestations affecting the skin, kidneys, central and peripheral nervous system and exocrine glands. We present the case of a 40-year-old man with left-sided ptosis, exophthalmos and headache. MRI demonstrated left-sided orbital pseudotumor with lacrimal and retro-orbital contrast enhancement extending to the cavernous sinus and the vestibulocochlear nerve. Immunological tests of serum and cerebrospinal fluid identified hepatitis $\mathrm{C}$ virus $(\mathrm{HCV})$ as a potential causative agent but did not indicate any additional infectious, malignant or immunological disorder. Hepatological evaluation revealed no signs of advanced liver disease. After initial spontaneous improvement, the patient subsequently developed vestibulocochlear failure with gait disorder, tinnitus and transient left-parietal sensory loss. Lacrimal biopsy demonstrated lymphocytic infiltrate, prompting steroid treatment. After initial improvement, steroids could not be tapered below $40 \mathrm{mg}$ daily for several months due to recurrent symptoms. Twelve months
\end{abstract}


after the initial presentation, the patient's chronic HCV infection was successfully treated with sofosbuvir, simeprevir and ribavirin and he remains now free of symptoms without steroids. In patients with chronic hepatitis $C$, lymphocytic infiltrate of the salivary and lacrimal glands is a frequent phenomenon. However, the extent of the lymphocytic infiltrate beyond the lacrimal gland to the tip of the orbit, cavernous sinus and vestibulocochlear nerve as in our patient is highly unusual. For all symptomatic extrahepatic manifestations of hepatitis $C$ infection, treatment of HCV as the underlying immune stimulus is recommended, and it helped to control the symptoms in our patient. In addition, long-term follow-up for recurrent lymphocyte infiltrate and development of lymphoma is warranted.

(C) 2016 The Author(s)

Published by S. Karger AG, Basel

\section{Introduction}

Chronic hepatitis $\mathrm{C}$ virus (HCV) infection remains the most important cause of chronic liver disease in Western countries and in the developing world. The seroprevalence of hepatitis $\mathrm{C}$ has been estimated to be at $2.8 \%$ worldwide [1]. Following infection, the majority of individuals (54-86\%) do not clear the virus and develop chronic hepatitis C infection, which without treatment remains a lifelong chronic condition. These individuals are at risk of developing chronic liver disease. Progression of HCV liver disease is slow, with 4-24\% patients developing liver cirrhosis within a time frame of 20-30 years [2]. HCV cirrhosis carries the risk of hepatic decompensation in $2-5 \%$ of patients per year and liver cancer in $1-4 \%$ of patients per year. HCV infection is the most frequent etiology of chronic liver disease in patients on waiting lists for liver transplantation, illustrating the magnitude of the problem [3].

In up to $74 \%$ of HCV patients, extrahepatic manifestations have been described, leading to a significant impairment in quality of life even in patients with only mild liver disease [4]. Fatigue is the most frequently reported symptom (more than $50 \%$ of patients), followed by arthralgia, paresthesia, myalgia, pruritus and sicca syndrome [5]. Upon careful testing, mild cognitive impairment can be detected in a fraction of HCV-infected patients [6].

An association of HCV infection with immune dysfunction has also been demonstrated. Continuous antigen stimulation by chronic HCV infection can lead to clonal B-cell expansion and aberrant antibodies with rheumatoid factor activity and essential mixed cryoglobulinemia (MC) [7]. MC remains the most intensely studied extrahepatic manifestation of HCV.MC is associated with the deposition of circulating immune complexes in small- to medium-sized blood vessels, ultimately leading to systemic vasculitis. It is considered prelymphomatous, as approximately $10 \%$ of patients later develop lymphoma. Clinical manifestations include palpable purpura, arthralgia, asthenia, and organ involvement of skin, joints, kidney, nervous system, and salivary and lachrymal glands. More than $90 \%$ of patients suffering from essential MC are infected with HCV, and cryoglobulins can be detected in $50 \%$ of HCV patients, illustrating the association of both conditions [8].

HCV infection also has lymphotropic effects and can lead to an increased risk of B-cell disorders including monoclonal gammopathy and B-cell lymphoma [9]. In a large study of almost 150,000 HCV-infected patients, the risk of non-Hodgkin lymphoma was increased by $28 \%$ [10]. Similarly, in a large meta-analysis, the prevalence of HCV infection was approximately 10 times higher in patients with B-cell lymphoma than in the general population [11].

Further manifestations of HCV infection include rheumatological disorders such as arthritis (rheumatoid-like or MC-related) and Sjögren syndrome (SS), which manifests as xerostomia and xerophtalmia but is histologically different from primary SS [12]. HCV infection is 


\section{Case Reports in \\ Gastroenterology}

Case Rep Gastroenterol 2016;10:108-114

(c) 2016 The Author(s). Published by S. Karger AG, Basel www.karger.com/crg

Misselwitz et al.: Orbital Pseudotumor as a Rare Extrahepatic Manifestation of Hepatitis C Infection

associated with chronic lymphocytic sialadenitis, which is accompanied by the presence of $\mathrm{HCV}$ in the saliva in the majority of cases, and salivary gland lymphoma [13]. Kidney disease, most frequently MC-related, is commonly found in HCV-infected patients, and it is recommended that all individuals suffering from chronic kidney disease be tested for HCV infection [4]. Additionally, HCV-infected patients demonstrate higher levels of insulin resistance and carry an elevated risk for the development of diabetes mellitus [14].

HCV can also affect the central and peripheral nervous system [12]. Peripheral neuropathy, including mononeuropathy and polyneuropathy, has been described in HCV patients both with and without mixed cryoglobulins. HCV can also lead to cerebrovascular events including ischemic stroke and demyelinative disorder. Cerebrovascular events are typically mediated by MC vasculitis, but HCV has also been detected in cerebrospinal fluid (CSF), suggesting a more active role of the virus in some cases [15].

The recent introduction of direct acting antivirals (DAA) has tremendously improved treatment options for hepatitis $\mathrm{C}$, largely replacing interferon-based treatment with moderate efficacy and severe side effects. Treatment decreases the risk of liver cirrhosis, hepatic decompensation and hepatocellular carcinoma but has also been demonstrated to reduce lymphoma risk [16]. There is general consent that all HCV infections in patients with extrahepatic manifestations should be treated with high priority [17]. In the case of HCV-induced $\mathrm{MC}$, the recommended treatment is aggressive optimal antiviral therapy. Only in patients presenting with severe MC, immunosuppression has proved beneficial, combined with plasmapheresis in life-threatening cases [18]. However, less knowledge exists so far regarding treatment of extrahepatic manifestations of hepatitis C.

We present an unusual case of a patient with HCV infection who developed ptosis, orbital pseudotumor, vestibulocochlear involvement and lymphocytic dacryoadenitis.

\section{Case Presentation}

A 40-year-old man was referred to our ophthalmology clinic for left temporal and retroauricular pain, left-sided eyelid edema and ptosis (fig. 1a). Ophthalmological examination confirmed left-sided ptosis of $3 \mathrm{~mm}$ and a left-sided exophthalmos of $5 \mathrm{~mm}$. We also noted preexisting left-sided amblyopia and nystagmus due to myopia magna with an otherwise normal ophthalmic status, particularly normal pupillary reaction and eye motility. Physical and neurological examinations were normal. Therefore, a follow-up was scheduled without further treatment.

Six days later, the patient reported symmetric ocular pain and headaches associated with bilateral ptosis more pronounced on the left side. The patient was admitted as an inpatient. Laboratory testing revealed mild systemic inflammation (C-reactive protein $13 \mathrm{mg} / \mathrm{l}$ $(<5)$, leukocyte count $11.6 \mathrm{G} / \mathrm{l}(3-9.6)$, blood sedimentation rate $37 \mathrm{~mm}$ within the first hour and mild anemia [hemoglobin $123 \mathrm{~g} / \mathrm{l}(134-170)]$. CSF analysis revealed a cell count of $9 / \mu \mathrm{l}$ (0-4) with 77\% lymphocytes, normal glucose and a slightly elevated protein concentration (616 mg/dl; 200-500). Intrathecal IgA and IgM synthesis but no oligo- or monoclonal bands were detected in CSF or serum. Magnetic resonance imaging (MRI) of the brain and orbit showed contrast enhancement ranging from the tip of the left orbit to the cavernous sinus, the lacrimal gland and the superior rectus muscle (fig. 1b). The same regions also showed enhancement in a fluordeoxyglucose positron emission tomography with computed tomography (FDG-PET-CT). 
The patient history was remarkable for chronic HCV infection, but further testing for Treponema pallidum, Borrelia burgdorferi, tick-borne encephalitis, HIV and hepatitis B were negative. Microbiological, mycobacteriological and virological investigations of CSF were unremarkable with negative PCRs for cytomegalovirus, herpes simplex virus $1 / 2$ and varicella zoster virus. Tests for anti-nuclear antibodies (ANA), anti-neutrophil cytoplasmic antibodies (ANCA), anti-SSA/B, anti-Jo1 and anti-native DNA antibodies were negative, and all IgG subclasses (including IgG4) were within normal limits. With negative rheumatoid factor, cryoglobulins were not tested. Duplex ultrasound of extracranial vessels was unremarkable without evidence for vasculitis. Thoracic CT scan revealed a small granuloma in the right upper lobe but no findings suggestive for sarcoidosis.

The symptoms of the patient improved within 2 days without specific treatment with only mild left-sided headache and fatigue persisting. A previously scheduled biopsy of the lacrimal gland was cancelled, and the patient was discharged with frequent appointments for follow-up.

Six weeks after the initial presentation, vestibulocochlear failure occurred with imbalance, tendency to fall to the left, alternating left- and right-sided tinnitus and hearing loss mainly on the left side. A brief episode of left-parietal sensory loss was also noted. Reevaluation of the earlier MRI suggested discrete contrast enhancement of the left vestibulocochlear nerve. Due to progressive symptoms, biopsy of the lacrimal gland was performed. Histological examination revealed mixed lymphocyte infiltration with CD20-positive B cells and CD5-positive T cells (fig. 1c, d) and no evidence of increased IgG4-positive plasma cells. Molecular studies showed no clonal immunoglobulin rearrangement.

Treatment of lymphocytic dacryoadenitis with prednisone $80 \mathrm{mg} /$ day was started. Pain improved within 3 days; however, several trials to taper prednisone below $40 \mathrm{mg} /$ day within the following months prompted recurrent eye pain, headache, fatigue and tinnitus. MRI showed a strong reduction of inflammation on follow-up 8 months after presentation. At this time, the patient was still on prednisone $20 \mathrm{mg}$ /day with persistent mild retro-orbital pain and fatigue. The patient was referred to our hepatology unit for treatment of chronic HCV infection.

Hepatitis $\mathrm{C}$ genotype $1 \mathrm{~B}$ had been diagnosed 14 years earlier and the patient was in regular surveillance. Liver function tests were persistently normal. Viral load was 6.8 million $\mathrm{IU} / \mathrm{ml}$. Abdominal ultrasound on several occasions revealed hyperechogenic liver parenchyma, a lymph node in the liver hilum and a borderline spleen size (13 $\mathrm{cm}$ pole diameter). Transient elastography was normal ( $4.7 \mathrm{kPa})$. Treatment with sofosbuvir $400 \mathrm{mg} / \mathrm{d}$, simeprevir $150 \mathrm{mg} / \mathrm{d}$ and ribavirin $1,200 \mathrm{mg} / \mathrm{d}$ for 12 weeks was started. Therapy was well tolerated with no detectable virus 4 and 12 weeks after therapy consistent with a cure of hepatitis C infection.

During therapy, the patient's symptoms further improved and prednisone was tapered and finally stopped. Eighteen months after the initial presentation and 3 months after the end of HCV therapy, the patient remains free of symptoms without any treatment.

\section{Discussion}

We present a case of a patient with chronic HCV infection complicated by dacryoadenitis and orbital pseudotumor with symptomatic retro-orbital and vestibulocochlear involvement as a highly unusual extrahepatic manifestation of chronic hepatitis C. Inflammation of the lacrimal gland was due to infiltrating polyclonal $\mathrm{B}$ and $\mathrm{T}$ lymphocytes. The retro-orbital tis- 


\section{Case Reports in Gastroenterology}

Case Rep Gastroenterol 2016;10:108-114 $10.1159 / 000444011$

c) 2016 The Author(s). Published by S. Karger AG, Basel www.karger.com/crg

Misselwitz et al.: Orbital Pseudotumor as a Rare Extrahepatic Manifestation of Hepatitis C Infection

sue, supraorbital muscle and vestibulocochlear nerve showed simultaneous inflammation, most likely by an identical pathomechanism.

$\mathrm{HCV}$ has a tropism for lymphoid tissue, and HCV infection is frequently accompanied by activation and polyclonal expansion of B cells [9]. Glandular involvement is a frequent complication of hepatitis C, and lymphocyte infiltration due to HCV and primary SS shares similar histological features [19]. In a recent review, various xerostomia prevalence rates of up to $51 \%$ were reported in HCV patients (11\% in the largest study) [13]. In one illustrative study of $45 \mathrm{HCV}$ patients referred for liver biopsy, 23 patients described oral dryness and 20 patients ocular dryness in standardized questionnaires. Labial salivary gland biopsies were suggestive of SS in 21/45 patients [20]. Lacrimal glands are also frequently affected in HCV patients [13]. However, pseudotumor orbitae with ptosis and subsequent vestibulocochlear affection has, to the best of our knowledge, not been reported previously. We suggest that in our patient, lymphocytic infiltration did not stop at the lacrimal glands, but extended throughout the orbita and beyond, consistent with an extended presentation of glandular affection due to HCV.

Treatment options for hepatitis C dramatically improved with the introduction of DAAs, and for hepatitis $\mathrm{C}$ genotype 1 several highly efficient treatment options with no or negligible side effects have become available [21]. For these reasons, DAAs are preferred over traditional interferon-based treatment regimes. We used a combination of two DAAs and ribavirin to treat HCV infection. Interferon can occasionally cause dacryoadenitis [22, 23], similar to the lesions described in our patient, and was therefore considered contraindicated. There is general agreement that all symptomatic extrahepatic manifestations of hepatitis C should be treated $[7,17]$. Subsequently, after several failed attempts of steroid withdrawal with recurrent symptoms, successful treatment of HCV occurred concomitantly with complete resolution of symptoms and complete steroid withdrawal. In addition, eradication of hepatitis C might prevent lymphoma in the future which can complicate hepatitis C [24], SS [19] and also orbital pseudotumor by removing the stimulus for immune cell proliferation. Based on our past experience with patients with orbital pseudotumor in different clinical settings, we recommend follow-up of all patients with orbital pseudotumor and reevaluation if any changes and new symptoms occur.

Due to high costs, treatment of hepatitis $\mathrm{C}$ is currently limited by many health insurances to patients with advanced fibrosis/cirrhosis, but treatment of patients with symptomatic extrahepatic manifestations of hepatitis $C$ is usually covered. Therefore, recognition of such complications is also relevant for reimbursement of treatment costs in a given patient.

Our case report has several limitations. First, after cure of hepatitis $\mathrm{C}$ without reinfection no re-exposure to the antigen will ever occur, and by strict criteria an association of hepatitis $\mathrm{C}$ with the patient's symptoms has not been unanimously demonstrated in our case report. However, since lymphocyte expansion and glandular involvement are common phenomena in hepatitis $\mathrm{C}$, immune stimulation by HCV remains the most likely reason for the symptoms of our patient. Second, the symptoms of our patient continued to improve and steroid dosage was decreased during the first year after initial diagnosis, even before treatment of hepatitis C. We therefore do not know whether the clinical improvement concomitant with hepatitis $\mathrm{C}$ treatment was due to HCV treatment or just reflects the natural disease history in this patient.

In conclusion, we described lymphocyte dacryoadenitis with symptomatic retro-orbital and vestibulocochlear involvement in a patient with hepatitis $\mathrm{C}$. We propose pseudotumor orbitae due to retro-orbital lymphocytic inflammation as an uncommon presentation of 
lymphocytic infiltration of glandulae, which is a known extrahepatic manifestation of hepatitis C.

\section{Statement of Ethics}

The authors have not ethical conflicts to disclose. The patient provided written consent regarding publication of the clinical history and all images.

\section{Disclosure Statement}

Benjamin Misselwitz has received advisory fees from Gilead. Joachim Mertens has received travel funds from Gilead and AbbVie. Beat Müllhaupt has served as an advisory board member for Roche, MSD, Janssen Therapeutics, AbbVie, Boehringer Ingelheim, Gilead and BMS. In addition, he has served as consultant for Gilead and AbbVie and has received research grants from Roche and Gilead. Luc Biedermann, Michael Scharl, Eugenia Haralambieva, Andreas Lutterotti, Konrad P. Weber, Karla Chaloupka and Jana Epprecht declare no conflicts of interest.

\section{References}

1 Mohd Hanafiah K, et al: Global epidemiology of hepatitis C virus infection: new estimates of age-specific antibody to HCV seroprevalence. Hepatology 2013;57:1333-1342.

2 Seeff LB: Natural history of chronic hepatitis C. Hepatology 2002;36(5 suppl 1):S35-S46.

-3 Wong RJ, et al: Nonalcoholic steatohepatitis is the second leading etiology of liver disease among adults awaiting liver transplantation in the United States. Gastroenterology 2015;148:547-555.

4 Cacoub P, et al: Extrahepatic manifestations of chronic hepatitis C virus infection. Dig Liver Dis 2014;46(suppl 5):S165-S173.

5 Poynard T, et al: Fatigue in patients with chronic hepatitis C. J Viral Hepat 2002;9:295-303.

-6 Hilsabeck RC, Perry W, Hassanein TI: Neuropsychological impairment in patients with chronic hepatitis C. Hepatology 2002;35:440-446.

7 AASLD/IDSA HCV Guidance Panel: Hepatitis C guidance: AASLD-IDSA recommendations for testing, managing, and treating adults infected with hepatitis C virus. Hepatology 2015;62:932-954.

8 Zignego $\mathrm{AL}$, et al: Virus-driven autoimmunity and lymphoproliferation: the example of HCV infection. Expert Rev Clin Immunol 2015;11:15-31.

9 Ferri C, et al: Hepatitis C virus syndrome: a constellation of organ- and non-organ specific autoimmune disorders, B-cell non-Hodgkin's lymphoma, and cancer. World J Hepatol 2015;7:327-343.

10 Giordano TP, et al: Risk of non-Hodgkin lymphoma and lymphoproliferative precursor diseases in US veterans with hepatitis C virus. JAMA 2007;297:2010-2017.

11 Gisbert JP, et al: Prevalence of hepatitis C virus infection in B-cell non-Hodgkin's lymphoma: systematic review and meta-analysis. Gastroenterology 2003;125:1723-1732.

12 Tampaki M, Koskinas J: Extrahepatic immune related manifestations in chronic hepatitis C virus infection. World J Gastroenterol 2014;20:12372-12380.

$\checkmark 13$ Carrozzo M: Oral diseases associated with hepatitis C virus infection. Part 1. Sialadenitis and salivary glands lymphoma. Oral Dis 2008;14:123-130.

-14 Negro F, et al: Extrahepatic morbidity and mortality of chronic hepatitis C. Gastroenterology 2015;149:1345-1360.

15 Mouelhi L, et al: Transverse myelitis associated with chronic viral hepatitis C. Tunis Med 2010;88:116118.

-16 Kawamura Y, et al: Viral elimination reduces incidence of malignant lymphoma in patients with hepatitis C. Am J Med 2007;120:1034-1041.

-17 European Association for Study of Liver: EASL Recommendations on treatment of hepatitis C 2015. J Hepatol 2015;63:199-236. 


\section{Case Reports in \\ Gastroenterology}

\begin{tabular}{l|l}
\hline Case Rep Gastroenterol 2016;10:108-114 \\
\hline $10.1159 / 000444011$ & $\begin{array}{l}\text { ○ 2016 The Author(s). Published by S. Karger AG, Basel } \\
\text { www.karger.com/crg }\end{array}$ \\
\hline
\end{tabular}

Misselwitz et al.: Orbital Pseudotumor as a Rare Extrahepatic Manifestation of Hepatitis C Infection

18 Cacoub P, Terrier B, Saadoun D: Hepatitis C virus-induced vasculitis: therapeutic options. Ann Rheum Dis 2014;73:24-30.

19 Ramos-Casals M, et al: Primary Sjögren syndrome: hematologic patterns of disease expression. Medicine (Baltimore) 2002;81:281-292.

20 Loustaud-Ratti V, et al: Prevalence and characteristics of Sjögren's syndrome or Sicca syndrome in chronic hepatitis C virus infection: a prospective study. J Rheumatol 2001;28:2245-2251.

21 Pawlotsky JM, et al: From non-A, non-B hepatitis to hepatitis C virus cure. J Hepatol 2015;62(1 suppl):S87-S99.

22 Morley AM, et al: Sarcoid-related dacryoadenitis following treatment with interferon alpha and ribavarin for hepatitis C. Orbit 2011;30:27-29.

23 Hwang, CJ, Gausas RE: Sarcoid-like granulomatous orbital inflammation induced by interferon-alpha treatment. Ophthal Plast Reconstr Surg 2008;24:311-313.

24 Paydas S: Hepatitis C virus and lymphoma. Crit Rev Oncol Hematol 2015;93:246-256.
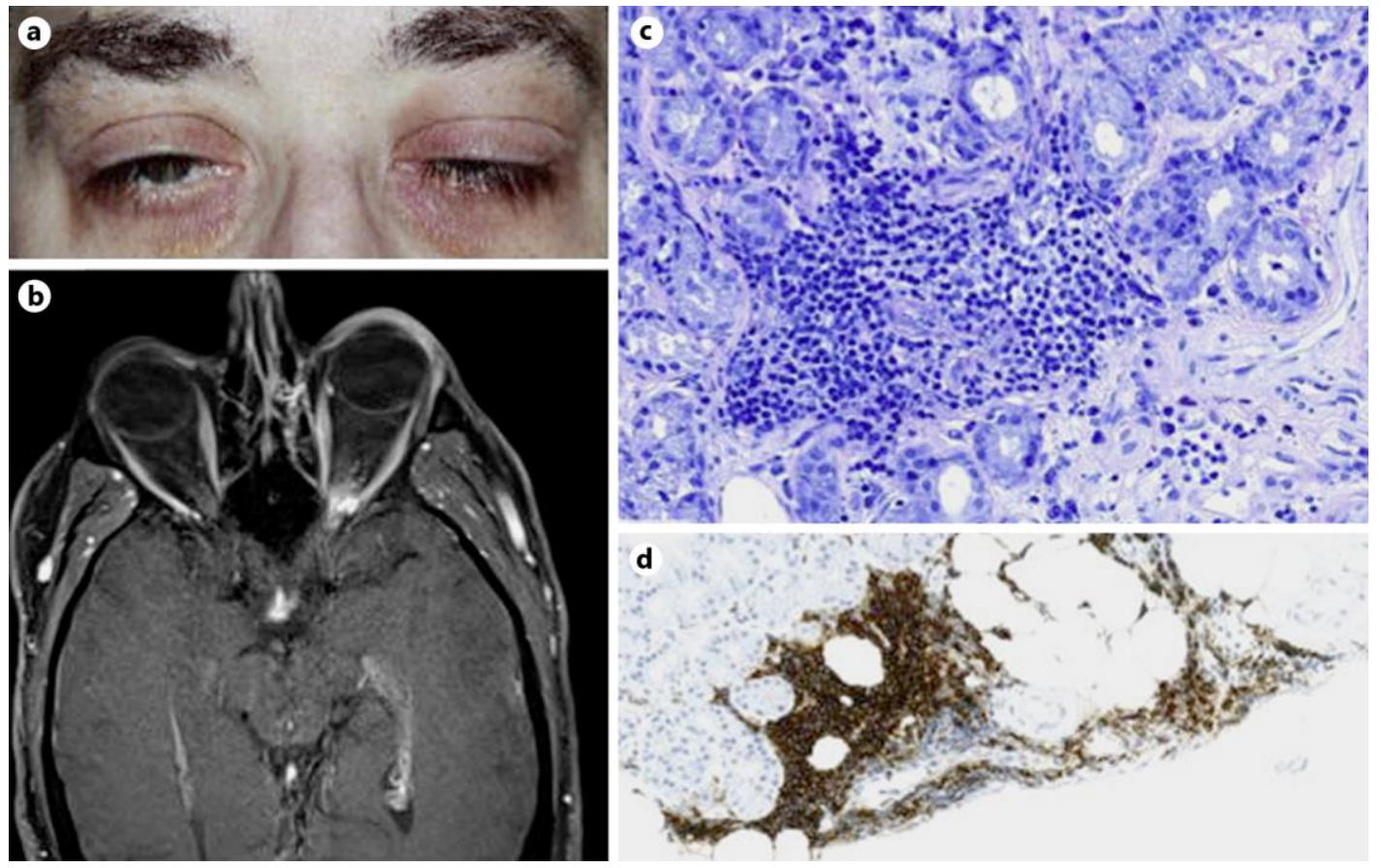

Fig. 1. Pseudotumor orbitae and lymphocytic dakryadenitis as rare extrahepatic manifestations of hepatitis C. a Presentation of our patient with bilateral ptosis on the left side more than on the right. $\mathbf{b}$ MRI of the brain and orbit (T1-weighted contrast-enhanced image) demonstrating left-sided pseudotumor orbitae. c Lacrimal gland with a prominent lymphocytic infiltration. Giemsa. $\times 200$. d Abundant CD20-positive B cells. CD20. $\times 100$. 